\title{
Quiescent Burst Evidence for Two Distinct GRB Emission Components
}

\author{
Jon Hakkila and Timothy W. Giblin \\ Department of Physics and Astronomy, College of Charleston, Charleston, SC 29424-0001
}

\begin{abstract}
We have identified two quiescent GRBs (bursts having two or more widelyseparated distinct emission episodes) in which the post-quiescent emission exhibits distinctly different characteristics than the pre-quiescent emission. In these two cases (BATSE GRBs 960530 and 980125), the second emission episode has a longer lag, a smoother morphology, and softer spectral evolution than the first episode. Although the pre-quiescent emission satisfies the standard internal shock paradigm, we demonstrate that the post-quiescent emission is more consistent with external shocks. We infer that some observed soft, faint, long-lag GRBs are external shocks in which the internal shock signature is not observed. We further note that the peak luminosity ratio between quiescent episodes is not in agreement with the ratio predicated by the lag vs. peak luminosity relationship. We briefly discuss these observations in terms of current collapsar jet models.
\end{abstract}

Subject headings: gamma rays: bursts - methods: data analysis

\section{Introduction}

There is strong evidence that the majority of gamma-ray burst (GRB) pulses result from internal shocks in relativistic winds (Sari \& Piran 1997a; Kobayashi, Piran, \& Sari 1997; Daigne \& Mochkovitch 1998; Ramirez-Ruiz \& Fenimore 2000; Nakar \& Piran 2002). However, a number of bursts exhibit a soft component indicative of external shocks that could be interpreted as onset of afterglow (Giblin et al. 1999; Burenin et al. 1999; Giblin et al. 2002). This emission begins preferentially towards the end of the burst or even after the GRB has ended (as suggested by the BeppoSAX WFC and NFI observations of serveral x-ray afterglows (Costa 2000)), but can also appear at a time early enough to overlap the shorttimescale emission (as observed in GRB 980923 (Giblin et al. 1999)). In addition, extended soft $\gamma$-ray emission has been observed by co-adding the fluxes of many BATSE bursts; this might also be an indicator of the same external shock phenomenon (Connaughton 2002). 
"Quiescent" GRBs may provide new clues to the origins and physics of GRB shocks. Quiescent bursts are GRBs that release their gamma-ray energy in more than one distinct emission episode (Ramirez-Ruiz \& Merloni 2001; Ramirez-Ruiz, Merloni, \& Rees 2001); e.g. they have at least one extended period during which emission is absent. A rigorous definition of quiescent time can be problematic due to the diverse nature or complexity of GRB temporal structures.

Ramirez-Ruiz \& Merloni (2001) introduced a quiescent GRB definition for a sample of bright BATSE bursts that used a sliding temporal window with a width of $0.05 \times$ T90 on summed, background-subtracted, BATSE 4-channel 64-ms data. These authors chose GRBs which had at least one time period for which the count rate dropped below $2 \sigma$ of the summed background rate. This criterion was applied to only the brightest, longest bursts in the BATSE 4B Catalog (Paciesas et al. 1999).

It is possible that this definition is too permissive, since it might allow for inclusion of some GRBs that do not have distinct emission episodes. For example, some bright bursts that do not satisfy this criterion (because the first episode's decay and the second episode's rise overlap) might be classified as quiescent if they are observed at lower signal-to-noise, as might some bursts with faint, long, underlying pulses. Because the existing quiescent definition might be too permissive, we have tentatively further required quiescent GRBs to be those in which the emission drops to the background for an extended period of time that is greater than or equal to the duration of the previous emission episode. This ensures that the quiescent time is greater than the interpulse duration.

In their study of very bright quiescent GRBs, Ramirez-Ruiz \& Merloni (2001) operated under the assumption that the post-quiescent episodes are due to internal shocks. We offer the hypothesis that quiescent GRBs potentially provide a laboratory in which external shock

signatures indicative of the onset of afterglow may be isolated from the rest of the burst. We have thus far found two GRBS that support this hypothesis.

\section{Analysis}

GRB 960530 and GRB 980125 are bursts from the BATSE 5B catalog that deviate significantly from what is considered to be standard GRB behavior. Neither burst was observed by Bepposax, and the RXTE PCA was unable to localize GRB 980125.

The BATSE 4-channel time histories of GRB 960530 and GRB 980125 are illustrated in figure 1 and figure 2. We have measured quiescent and emission episodic durations for these GRBs via the summed four-channel sliding temporal window technique of Ramirez-Ruiz \& 
Merloni (2001). As shown below, the localization measurements verify that the multiple emission episodes are indeed associated with the same sources for both GRB 960530 and GRB 980125.

GRB 960530 (BATSE trigger 5478) has two distinct emission episodes separated by 233 s. Episode (a) is a single-pulse FRED (Fast Rise Exponential Decay) of duration 23 s. Episode (b) is a smooth single-pulse episode of $37 \mathrm{~s}$. The BATSE locations of these episodes are consistent with the same source; episode (a) is located at $\mathrm{RA}=143.0^{\circ}$ and $\mathrm{Dec}=-53.8^{\circ}$ with error $\pm 1.4^{\circ}$, while episode (b) is located at $\mathrm{RA}=143.0^{\circ}$ and $\mathrm{Dec}=-53.0^{\circ}$ with error $\pm 1.9^{\circ}$.

GRB 980125 (BATSE trigger 6581) has two distinct emission episodes with a faint preburst episode. The preburst episode is faint and lasts $13 \mathrm{~s}$. It is followed by a short quiescent period of $18 \mathrm{~s}$. Although the preburst episode satisfies our rigorous definition of a distinct emission episode, we have chosen not to include it in our analysis because it is too faint for its properties to be accurately measured. Complex emission episode (a) follows and lasts $31 \mathrm{~s}$. Episode (b) is a smooth episode with two distinct pulses and a duration of roughly $45 \mathrm{~s}$, it is preceded by a quiescent period of $161 \mathrm{~s}$. The first of these two distinct pulses is a smooth broad pulse similar in temporal structure to GRB 960530's emission episode (b), while the second pulse (which overlaps the first) is more indicative of a standard FRED. The BATSE locations of these episodes are also consistent with the same source; the faint preburst pulse is located at $\mathrm{RA}=344.2^{\circ}$ and $\mathrm{Dec}=41.0^{\circ}$ with an error of $\pm 3.8^{\circ}$, emission episode (a) is located at $\mathrm{RA}=350.6^{\circ}$ and $\mathrm{Dec}=35.1^{\circ}$ with an error of $\pm 0.5^{\circ}$, and emission episode $(\mathrm{b})$ is located at $\mathrm{RA}=354.6^{\circ}$ and $\mathrm{Dec}=34.8^{\circ}$ with error $\pm 1.4^{\circ}$.

We demonstrate that the smooth quiescent pulses have other peculiar properties in addition to their peculiar temporal properties. To study these quiescent GRBs in more detail, we apply three techniques: (1) spectral lags, (2) Color-Color Diagrams (CCDs), and (3) the Internal Luminosity Function (ILF).

\subsection{Spectral Lags}

Spectral lags are energy-dependent delays in the GRB temporal structure. In general, soft pulses lag behind hard pulses. Pulses also appear to broaden at softer energies as $E^{-0.4}$ (Norris et al. 1996). Spectral lags apparently correlate with burst parameters such as beaming angles and peak luminosities (as measured on the $256 \mathrm{~ms}$ timescale) (Norris 2002).

Spectral lags are defined as the maximum value of the Cross-Correlation Function (CCF) of two energy channels (Band 1997). Poisson background variations in the time histories 
complicate the identification of the CCF peak, so prior studies have modeled the CCF with a fitting function to identify the lag. CCFs are typically asymmetric because GRBs generally have longer decay times than rise times. A cubic fitting function has been found to be a more accurate characterization of CCF lags (Norris, Marani, \& Bonnell 2000) than a quadratic function (Wu \& Fenimore 2000).

Rather than use a cubic or quadratic fitting function, we have instead chosen to use the pulse model of Norris et al. (1996). This function is a more natural choice than the cubic or quadratic since the CCF describes a normalized comparison between episodes whose primary structures are pulses; it thus accounts for the CCF time-asymmetry in a natural way.

Application of the CCF to the different emission components of GRB 960530 and GRB 980125 leads to intriguing results. The smooth pulse appearing after the quiescent period in GRB 960530 has a significantly longer lag (in all energy channel combinations) than the first FRED pulse. The lags are shown in table 1. The results are similar and are shown in table 2) for GRB 980125: the smooth pulse in emission episode (b) has a broader CCF and a significantly longer lag than that found during the rest of the burst. It should be noted that the lag of emission episode (a) is consistent with the lag of the second (FRED) pulse found in emission episode (b).

\subsection{The Internal Luminosity Function}

The Internal Luminosity Function (ILF) $\psi(L)$ is the distribution of luminosity within a GRB; $\psi(L) \Delta L$ represents the fraction of time during which a burst's luminosity lies between $L$ and $L+\Delta L$ (Horack \& Hakkila 1997; Hakkila et al. 2004). For the purposes of this manuscript, we discuss the ILF calculated in the 50 to $300 \mathrm{keV}$ energy range using fourchannel 64-ms data. The ILF is normalized to the number of Poisson background-subtracted time intervals during which emission is observed $2 \sigma$ or more above the background and by the requirement that $\Sigma \psi(L) \Delta L=1$. It is best fit with a quasi power-law form for most bursts such that

$$
\psi(L) \propto L^{\alpha} \times 10^{\beta \times[\log L]^{2}} .
$$

We refer to $\alpha$ as the power-law index and $\beta$ as the curvature index. The variables $\alpha$ and $\beta$ appear to be indicators of burst temporal morphology (they are highly-correlated such that GRBs with large $\alpha$ also have large $\beta$ while GRBs with small $\alpha$ also have small $\beta$ ); smooth, FRED-like bursts typically have large $\alpha$ (roughly $-2 \leq \alpha<3$ ) while spiky (narrow-pulsed) bursts typically have small $\alpha$ (approximately $-7 \leq \alpha<-2$ ).

We have measured the ILF for the different components of quiescent GRBs 960530 and 
980125; these are indicated in table 1 and table 2. We note that the late-appearing, smooth, long-lag components of both bursts are fit by noticeably steeper ILF values than are the other episodes. The ILF values of these smooth pulses thus indicate a different morphology than that normally associated with FREDs. The ILF thus supports the hypothesis that the smooth broad pulses are different in nature than the pulses found elsewhere in these two quiescent GRBs.

The second (FRED) pulse in emission episode (b) (at time t=255 s) of GRB 980125 is somewhat peculiar. It is smoother than a typical FRED and has a correspondingly steep value of $\alpha$, but it has a short lag consistent with the burst's emission episode (a). It is possible that overlap with the broad, long-lag pulse has affected its ILF measurement.

\subsection{Color-Color Diagrams}

Color-color diagrams (CCDs) (Giblin 2000) can be used to describe GRB spectral evolution. Several colors can be created from BATSE four-channel data; Giblin (2000) defines the hard color index $\mathrm{HC}=F 3 / F 2$ where $\mathrm{F} 3$ is the BATSE channel 3 photon flux in units of photons $\mathrm{cm}^{-2} \mathrm{~s}^{-1}$ and F2 is the BATSE channel 2 photon flux. The soft color index SC is defined similarly as $\mathrm{SC}=F 2 / F 1$ where $\mathrm{F} 1$ is the BATSE channel 1 photon flux. The timescales over which these color indices are measured vary in order to maintain a roughly constant signal-to-noise ratio. The BATSE color indices in general have large enough signal-to-noise ratios for reliable spectral evolution studies. Given the detector response, the observed colors of cooling external shocks have been predicted by Giblin (2000). With this information, CCDs can be used to search GRBs for signatures of external shocks. Having ascertained that the temporal and temporally-shifted spectral structures of the broad pulses are different than those of typical GRB pulses, we now explore the spectral evolution of these pulses using CCDs.

GRBs typically undergo hard to soft spectral evolution. Thus, the terminal GRB emission tends to be softer than the initial emission. This general spectral evolution can be observed in a CCD.

Although the emission appearing towards the end of GRBs tends to be soft, not all terminal emission exhibits the colors predicted by the external shock model. Theoretical synchrotron cooling models are predicted to occupy a fairly narrow range of hard vs. soft colors. Both the fast cooling and slow cooling models are predicted to have their color indices evolve in the regimes $0.6 \leq S C \leq 1.0$ and $0.7 \leq H C \leq 1.1$ (Giblin 2000; Giblin et al. 2002).

GRB 960530 has previously been considered to be an afterglow candidate because it 
exhibits a soft gamma-ray component that was coincident with the onset of the afterglow phase (Giblin et al. 2002). The CCD of this soft gamma-ray component was additionally in agreement with the theoretical prediction for synchrotron cooling. It is difficult to firmly establish the connection between the CCD of late emission from GRB 980125 and the synchrotron shock model, but figure 3 indicates that this late emission is consistent with both fast- (solid line), and slow-cooling (dashed line) synchrotron models. Furthermore, the second episode is in better agreement with synchrotron afterglow emission than the first episode (Freismuth, Giblin, \& Hakkila 2003).

The concluding time periods of GRB 960530 and GRB 980125 thus both exhibit spectral behavior that evolves from hard to soft (such as is typically found in GRBs). However, this behavior is also consistent with a particular spectral evolution expected from external shocks initiating an afterglow.

\section{Discussion}

The late, long-lag pulses of quiescent GRBs 960530 and 980125 have different temporal and spectral characteristics than those found earlier in the bursts. More notably, the properties are also different than those generally observed in GRBs. These properties indicate a separate pulse component which has been conveniently isolated from the standard emission in these two GRBs by the quiescent interval.

\subsection{Are the Long-Lag Components Internal or External Shocks?}

Despite the evidence linking GRB pulses to internal shocks, the long-lag pulses of GRBs 960530 and 980125 appear to be inconsistent with internal shocks. As can be seen in figure 1 and figure 2, the long-lag pulses appearing late in the bursts are broader than the short-lag pulses appearing early in the bursts, as expected from external shock models (Fenimore, Madras, \& Nayakshin 1996; Sari \& Piran 1997b; Fenimore et al. 1999; Fenimore \& RamirezRuiz 1999). We thus suspect that this newly-identified emission component is an external shock signature.

Further evidence supporting the external shock hypothesis can be found from the relationship observed between the duration of the post-quiescent emission episode and the quiescent time (Ramirez-Ruiz \& Merloni 2001; Ramirez-Ruiz, Merloni, \& Rees 2001). These authors found a linear, one-to-one ratio which they interpreted as a metastable configuration in the outflow such that a significant energy fraction still stored in the fireball is available for 
liberation through additional internal shocks at a later time. Therefore a longer quiescent interval will be followed by a longer post-quiescent emission episode. GRB 960530 and GRB 980125 do not obey this relation. In GRB 960530, the ratio of the post-quiescent emission episode duration to the quiescent time is $37 \mathrm{~s} / 233 \mathrm{~s}=0.16$, while in GRB 980125 the ratio is $45 \mathrm{~s} / 161 \mathrm{~s}=0.28$. These values deviate considerably from those considered "standard" for quiescent GRBs. Because these values are not as expected for internal shocks, they could be examples of external shocks.

The newly-identified long-lag pulses have been found to occur towards the end of these GRBs; however, we note that the long-lag pulse in GRB 980125 occurred prior to an additional concluding FRED pulse. (NOTE: both quiescent pulses have nearly the same peak flux.) The long-lag component may be present at even earlier times in other bursts. If so, then the newly-identified pulse type may be difficult to isolate because it is smooth, broad, and faint: it could overlap with narrower internal shock pulses to create complex temporal structures and complex spectral evolution. We note that many GRBs can be characterized by complex combinations of overlapping broad and narrow pulses.

\subsection{Impact on the Lag vs. Peak Luminosity Relation}

The lag vs. peak luminosity relation (Norris, Marani, \& Bonnell 2000) is

$$
L_{53} \approx 1.3 \times \tau^{-1.14}
$$

where $\tau=\left(\operatorname{lag}_{31} / 0.01 \mathrm{~s}\right), \operatorname{lag}_{31}$ is the lag between the $100-300 \mathrm{keV}$ channel and the 25-50 $\mathrm{keV}$ channel, and $L_{53}$ is the isotropic peak luminosity on the $256 \mathrm{~ms}$ timescale in units of $10^{53} \mathrm{erg} \mathrm{s}^{-1}$. The relation was originally calibrated for a half-dozen GRBs of known redshift. However, the anomalously-underluminous long-lag GRB 980425 was not included in the calibration.

The long-lag emission pulses of GRBs 960530 and 980125 provide a dilemma for the lag vs. peak luminosity relation, since that relation implicitly assumes that each GRB can be characterized by a single lag. However, very long post-quiescent lags do not put the relation at risk since there are normal bursts having even longer lags. According to figure 2 of Norris (2002), roughly 300 individual GRBs have $\operatorname{lag}_{21}>0.43 \mathrm{~s}$, where $\operatorname{lag}_{21}$ is the lag between the $50-100 \mathrm{keV}$ channel and the $25-50 \mathrm{keV}$ channel $\left(\operatorname{lag}_{21}=1.17 \mathrm{~s}\right.$ for GRB 960530 and

$\operatorname{lag}_{21}=0.42 \mathrm{~s}$ for GRB 980125). The long post-quiescent lags of GRBs 960530 and 980125 are common enough that they appear to represent a normal GRB behavior.

It is possible that some bursts exhibit only the smooth, long lag (external shock) component. The variable, short lag (internal shock) component would most likely be unde- 
tected in these bursts. There is a distinct possibility, then, that Norris (2002) has applied the lag vs. peak luminosity relation to bursts dominated by short-lag components as well as to potentially different bursts dominated by long-lag components. Quiescent GRBs 960530 and 980125 can provide a consistency check for GRBs that exhibit both behaviors. One would expect the post-quiescent to pre-quiescent luminosity ratios of these GRBs asobtainedfromthelagmeasurements to be equal to their peak flux ratios.

For pre-quiescent episode $a$ and post-quiescent episode $b$, the lag vs. peak luminosity relation predicts a ratio of peak luminosities

$$
\frac{L_{b}}{L_{a}}=\left(\frac{\tau_{b}}{\tau_{a}}\right)^{-1.14}
$$

For GRB 960530, $\tau_{\mathrm{b}} / \tau_{\mathrm{a}}=8.7$, from which $L_{\mathrm{b}} / L_{\mathrm{a}}=0.065$. However, the ratio of $256 \mathrm{~ms}$ peak fluxes gives $P_{\mathrm{b}} / P_{\mathrm{a}}=L_{\mathrm{b}} / L_{\mathrm{a}}=0.46$. For GRB 980125, $\tau_{\mathrm{b}} / \tau_{\mathrm{a}}=0.048$, which predicts $L_{\mathrm{b}} / L_{\mathrm{a}}=0.024$. The ratio of $256 \mathrm{~ms}$ peak fluxes is $P_{\mathrm{b}} / P_{\mathrm{a}}=L_{\mathrm{b}} / L_{\mathrm{a}}=0.15$.

The peak luminosities of the short-lag (a) and long-lag (b) components are plotted in figure 4 together with the six short-lag GRBs (filled diamonds) used to calibrate the lag vs. peak luminosity relation (Norris, Marani, \& Bonnell 2000). The luminosities of the long-lag components are predicted using both the lag vs. peak luminosity relation and peak flux ratios; the values predicted by the lag vs. peak luminosity relation lie on the theoretical (dashed) line while those predicted from peak flux ratios are shifted vertically from this line. The luminosities calculated from peak fluxes are larger than those calculated from the lag vs. peak luminosity relation, suggesting that the luminosities of these long-lag components are overluminous relative to the lag vs. peak luminosity relation.

This is difficult to reconcile with long-lag GRB 980425, which is underluminous relative to the lag vs. peak luminosity relation (see figure 4) rather than overluminous. Even though GRB 980425 and the post-quiescent emission of GRBs 960530 and 980125 all have long lags of similar durations, it appears that these lags are not good predictors of peak luminosity. The long-lag GRBs do not all obey the same lag vs. peak luminosity relation as short-lag GRBs, and it is possible that they do not obey a lag vs. peak luminosity relation at all.

\subsection{How Common is the Long-Lag Emission Component?}

The long-lag emission component is likely common in many GRBs, even though it has, thus far, only been expicitly identified in only two quiescent GRBs. The lag calculations of Norris, Marani, \& Bonnell (2000) use an apodization technique to remove low-intensity emission from the lag calculation because a long-lag signature hidden in the low-intensity 
emission is capable of distorting the lag measurement (e.g. GRB 990123). The authors thus implicitly recognize the presence of a long-lag component arising from low-intensity emission. In addition to this signature, we have seen evidence of additional long-lag emission in the decaying tail of GRB 991216 (BATSE trigger 7906). As indicated by Norris (2002), it is also possible that many GRBs consist of a long-lag component only.

\subsection{Impact on Theoretical Models}

The two different pulse types observed here presumably arise from the same collimated relativistic outflow. The narrow pulses originate from colliding shells in the high- $\Gamma$, tightlycollimated jet center (e.g. Mészáros, Rees, \& Wijers (1998); Kumar \& Piran (2000)), where $\Gamma$ is the bulk Lorentz factor. The external shocks initiating the onset of afterglow are produced by a low- $\Gamma$, slowly-expanding shock found at the interface between the jet and the external medium (i.e. the external shock). The opening angle of the jet should increase

as deceleration sets in (e.g. Ramirez-Ruiz \& Fenimore (1999)). The detailed structure of GRB relativistic jets is still unclear, but determination of this structure is currently being performed using numerical hydrodynamic codes (e.g. (Zhang, Woosley, \& MacFadyen 2003; Aloy et al. 2003)). In fact, hydrodynamic simulations indicate that the beaming angle of the high- $\Gamma$ component is limited to $\sim 5^{\circ}$, whereas the low- $\Gamma$ component can have a beaming angle exceeding $15^{\circ}$ Zhang, Woosley, \& MacFadyen (2003). The angular expansion of the external shock can be aided by the presence of a "cocoon jet", which is a secondary jet structure that forms when energy is deposited in a stellar cavity prior to breakout Ramirez-Ruiz, Celotti, \& Rees (2002).

In the standard internal/external shock model, deceleration of the relativistic ejecta sets in at some deceleration distance $R_{d e c} \sim \Gamma^{-2 /(3-s)}$ in the surrounding environment with density $n(r) \sim r^{-s}$, resulting in a temporal delay between the prompt internal shock $\gamma$-ray emission and the afterglow (Ramirez-Ruiz, Celotti, \& Rees 2002). Thus, for a given density profile, large $\Gamma$ yields a small $R_{d e c}$. A temporal overlap between the internal and external shock emission would be expected in these cases, producing complex $\gamma$-ray light curves. The optical flash that occurred some $\sim 40$ s into the prompt $\gamma$-ray emission of GRB 990123 has been interpreted as deceleration during the burst due to the reverse external shock (Sari \& Piran 1999; Mészáros, \& Rees 1999; Soderberg \& Ramirez-Ruiz 2002). Interestingly, the optical flash is temporally coincident with the long-lag component of GRB 990123. Sari \& Piran (1999) note that the x-ray emission peaked some $\sim 60$ s into the burst. This overlaps in time with the long-lag emission, suggesting that the long-lag component may represent the onset of the afterglow, e.g. the long-lived forward shock emission. On the other hand, the 
low-intensity long-lag emission exhibits some short timescale variability. Fenimore, RamirezRuiz, \& Wu (1999) demonstrated that the widths of these low-intensity pulses do not evolve with time, suggesting no deceleration and an internal shock origin. However, this does not preclude the possibility of narrow late-time pulses overlapping a broad long-lag emission component. We note that these behaviors are quite unlike the behaviors observed in that latter parts of GRBs 950530 and 980125 .

As noted before, the BATSE database contains many long-lag GRBs. These often have characteristics of FREDs, and many are initiated by what appear to be narrow, short-lag pulses. It is tempting to interpret these as GRBs produced by jets with small $R_{\text {dec }}$; few internal shocks are observed, and the onset of afterglow is thus the defining characteristic of their prompt emission. However, this hypothesis is complicated by the menagerie of other phenomena such as X-ray rich GRBs (XRRs), X-Ray Flashes(XRFs), and orphan afterglows, that are also thought to result from off-axis GRB viewing (e.g. Mészáros, Rees, \& Wijers (1998); Zhang et al. (2004)). This is because XRRs and XRFs are spectrally soft, but otherwise contain many pulses; some of these pulses are narrow and some are broad (R. Vanderspek, private communication). Our explanation of the long-lag bursts would imply that XRRs and XRFs are jets with small viewing angles (to explain the variable temporal structure) seen at large redshift, rather than being GRB jets viewed off-axis. However, Bloom et al. (2003) have shown that two recent XRFs originated at redshifts less than $z=3.5$.

\section{Conclusions}

At least two quiescent GRBs exhibit multiple lags. The short-lag component is consistent with internal shocks while an external shock explanation is preferred for the smooth long-lag component. The long-lag component is broad and faint, and is possibly present but unrecognizable in many bursts. The apodization of (Norris, Marani, \& Bonnell 2000) implicitly recognizes its existence in order to remove it. It is possible that many or all of the long-lag bursts in the sample of Norris (2002) exhibit only the smooth and broad long-lag component.

Because the short and long lags found in these quiescent GRBs are so different, we have assumed that there are two distinct GRB pulse types. This hypothesis needs to be verified. The best way to approach this problem is via statistical and/or data mining studies of GRB pulse characteristics. It is important to determine the existence of one continuous pulse distribution or poossibly distinctly different distributions. Detailed analyses of BATSE and Swift GRB data are planned to corroborate this work. 
The lag vs. peak luminosity relation apparently requires re-calibration. It is not clear that long-lag pulses obey the same relation as short-lag pulses, or if they even obey a lag vs. peak luminosity relation. Since there is a correlation between lag and variability, the variability vs. peak luminosity relation (Reichart et al. 2001) might require similar recalibration.

The simplest explanation for the large number of long-lag GRBs found in the BATSE database is that they are GRBs viewed from off the jet axis. The tightly-beamed, variable, short-lag internal shocks would not be seen from large viewing angles, whereas the more widely-beamed, smooth, long-lag external shocks would be. However, it is difficult to reconcile this explanation at the present time with some of the other similar phenomena associated with GRBs (e.g. x-ray rich GRBs, Gamma-Ray Flashes, and Orphan Afterglows).

\section{Acknowledgments}

We are grateful to the anonymous referee for a careful reading of this manuscript, and for suggestions that strengthened the theoretical discussion. We acknowledge R. D. Preece for calculating burst locations, and we are extremely grateful to T. Piran, E. E. Fenimore, E. Ramirez-Ruiz, J. Norris, J. Bonnell, and R. Vanderspek for valuable discussions. This research was sponsored by NASA grant NRA-98-OSS-03 and NSF grant AST00-98499.

\section{REFERENCES}

Aloy, M.-A., Martí, J.-M., Gómez, J.-L., Agudo, I., Müller, E. \& Ibanéz, J.-M. 2003, ApJ, 585, L109

Band, D. L. 1997, ApJ, 486, 928

Bloom, J. S., Fox, D., van Dokkum, P. G., Kulkarni, S. R., Berger, E., Djorgovski, S. G., \& Frail, D. A. 2003, ApJ, 599, 957

Burenin, R. A. et al. 1999, A\&A, 344, L53

Connaughton, V. 2002, ApJ, 567, 1028

Costa, E. 2000, AIP Conf. Proc. 526: Gamma-ray Bursts, 5th Huntsville Symposium, 365

Daigne, F. \& Mochkovitch, R. 1998, MNRAS, 296, 275

Fenimore, E. E., Madras, C. D., \& Nayakshin, S. 1996, ApJ, 473, 998 
Fenimore, E. E., Cooper, C., Ramirez-Ruiz, E., Sumner, M. C., Yoshida, A., \& Namiki, M. 1999, ApJ, 512, 683

Fenimore, E. E. \& Ramirez-Ruiz, E. 1999, ASP Conf. Ser. 190: Gamma-Ray Bursts: The First Three Minutes, 67

Fenimore, E. E., Ramirez-Ruiz, E., \& Wu, B. 1999, ApJ, 518, L73

Frail, D. A. et al. 2001, ApJ, 562, L55

Freismuth, T. M., Giblin, T., \& Hakkila, J. 2003, American Astronomical Society Meeting, 202

Giblin, T. W., van Paradijs, J., Kouveliotou, C., Connaughton, V., Wijers, R. A. M. J., Briggs, M. S., Preece, R. D., \& Fishman, G. J. 1999, ApJ, 524, L47

Giblin, T. W. 2000, Ph.D. Thesis

Giblin, T. W., Connaughton, V., van Paradijs, J., Preece, R. D., Briggs, M. S., Kouveliotou, C., Wijers, R. A. M. J., \& Fishman, G. J. 2002, ApJ, 570, 573

Hakkila, J., Giblin, T. W., Young, K. C., Fuller, S. P., Stallworth, A. D., \& Sprague, A. P., 2004, in proceedings of the 2003 Santa Fe Gamma-Ray Burst Conference (ed. E. E. Fenimore), submitted.

Horack, J. M. \& Hakkila, J. 1997, ApJ, 479, 371

Kobayashi, S., Piran, T., \& Sari, R. 1997, ApJ, 490, 92

Kumar, P. \& Piran, T. 2000, ApJ, 535, 152

Meegan, C. A. et al. 2003, Current BATSE Gamma-Ray Burst Catalog. Available at gammaray.msfc.nasa.gov/batse/grb/catalog

Nakar, E. \& Piran, T. 2002, ApJ, 572, L139

Mészáros, P., Rees, M. J. \& Wijers, R. A. M. J. 1998, ApJ, 499, 301

Mészáros, P., \& Rees, M. J. 1999, MNRAS, 306, L39

Norris, J. P. 2002, ApJ, 579, 386

Norris, J. P., Nemiroff, R. J., Bonnell, J. T., Scargle, J. D., Kouveliotou, C., Paciesas, W. S., Meegan, C. A., \& Fishman, G. J. 1996, ApJ, 459, 393 
Norris, J. P., Marani, G. F., \& Bonnell, J. T. 2000, ApJ, 534, 248

Norris, J. P., Scargle, J. D., \& Bonnell, J. T. 2001, AIP Conf. Proc. 587: Gamma 2001: Gamma-Ray Astrophysics, 176

Paciesas, W. S. et al. 1999, ApJS, 122, 465

Panaitescu, A. \& Kumar, P. 2002, ApJ, 571, 779

Ramirez-Ruiz, E., Celotti, A., \& Rees, M. J. 2002, MNRAS, 337, 1349

Ramirez-Ruiz, E. \& Fenimore, E. E. 2000, A\&AS, 138, 521

Ramirez-Ruiz, E. \& Fenimore, E. E. 2000, ApJ, 539, 712

Ramirez-Ruiz, E. \& Merloni, A. 2001, MNRAS, 320, L25

Ramirez-Ruiz, E., Merloni, A., \& Rees, M. J. 2001, MNRAS, 324, 1147

Reichart, D. E., Lamb, D. Q., Fenimore, E. E., Ramirez-Ruiz, E., Cline, T. L., \& Hurley, K. 2001, ApJ, 552, 57

Rees, M. J. \& Mészáros, P. 1994, ApJ, 430, L93

Sari, R. 1999, ApJ, 524, L43

Sari, R. \& Piran, T. 1997a, MNRAS, 287, 110

Sari, R. \& Piran, T. 1997b, ApJ, 485, 270

Sari, R. \& Piran, T. 1999, ApJ, 517, L109

Soderberg, A. M., \& Ramirez-Ruiz, E. 2002, MNRAS, 330, L24

Stern, B., Poutanen, J., \& Svensson, R. 1999, ApJ, 510, 312

Wu, B. \& Fenimore, E. 2000, ApJ, 535, L29

Zhang, B., Dai, X., Lloyd-Ronning, N. M., \& Mészáros, P. 2004, ApJ, 601, L119

Zhang, W., Woosley, S. E., \& MacFadyen, A. I. 2003, ApJ, 586, 356 


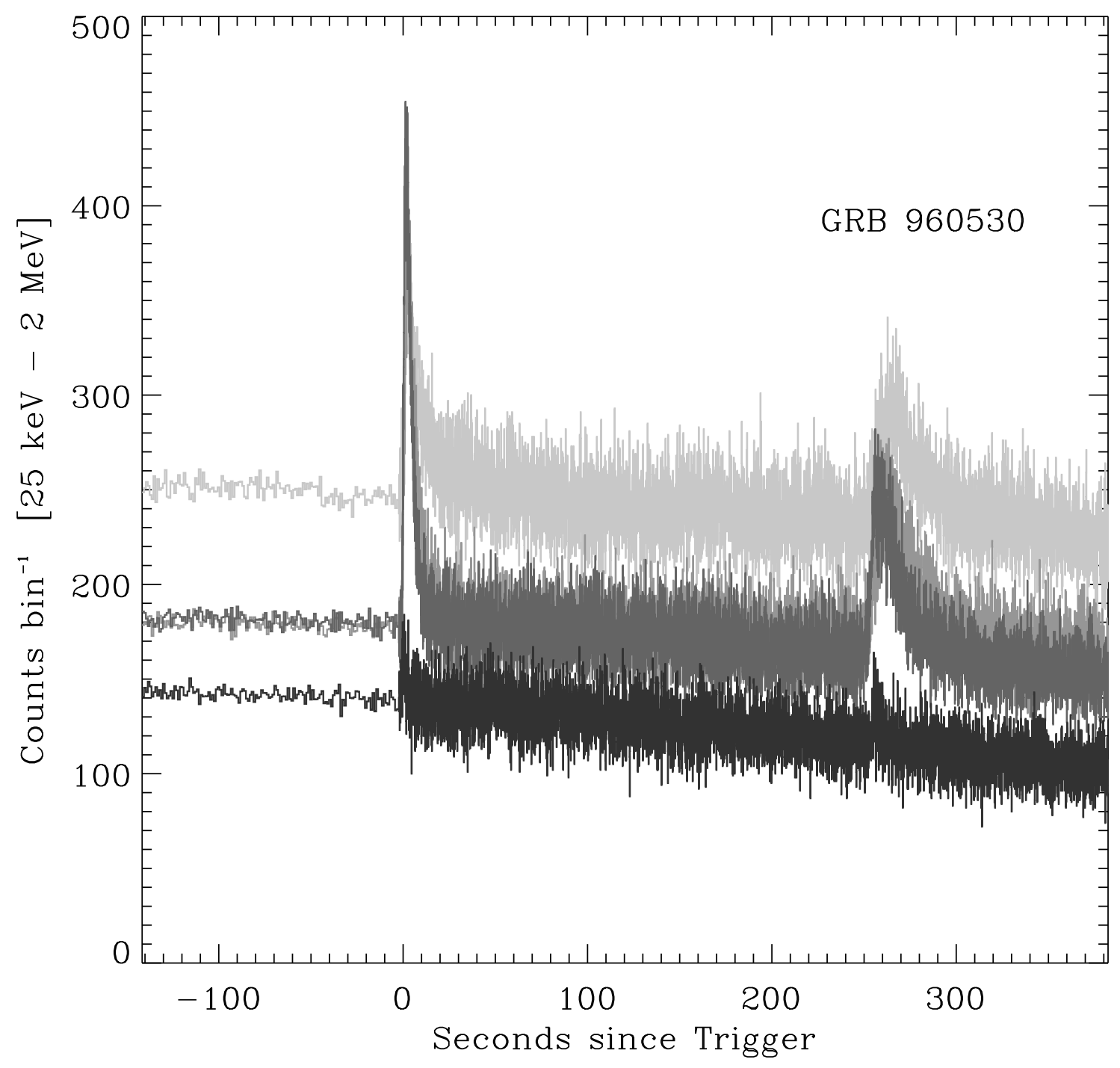

Fig. 1.- Four-channel time history of GRB 960530 (BATSE trigger 5478). 


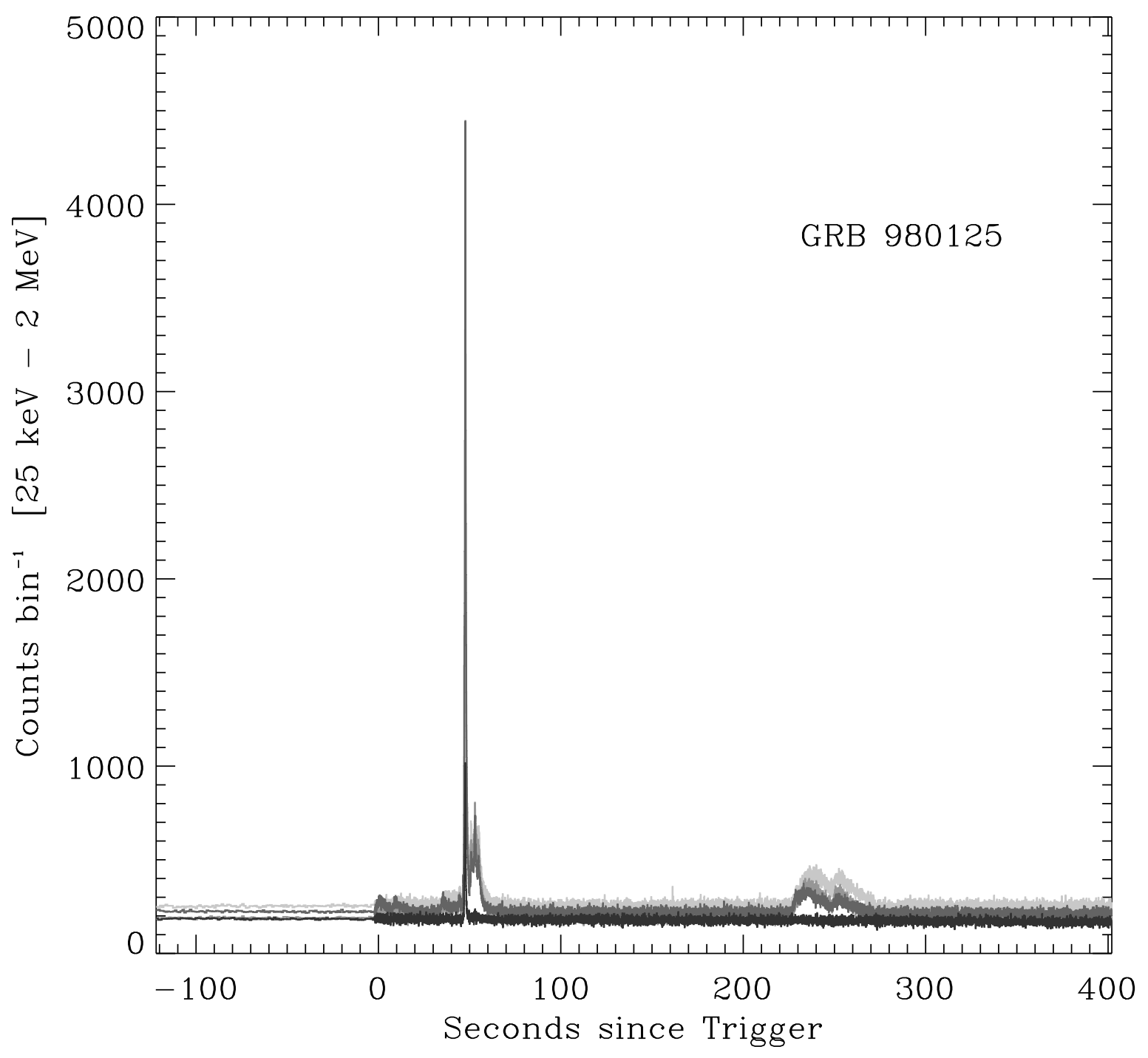

Fig. 2.- Four-channel time history of GRB 980125 (BATSE trigger 6581). 

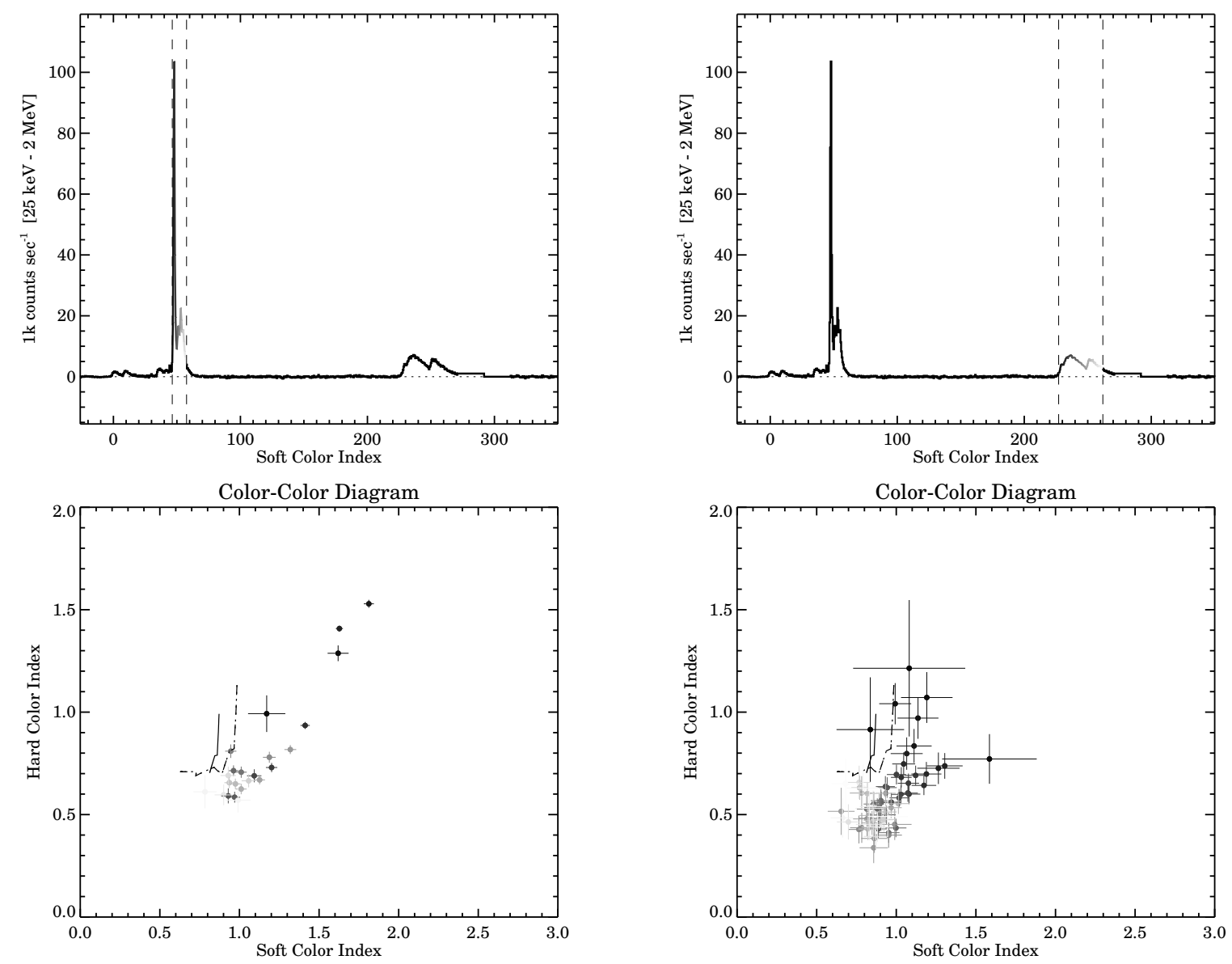

Fig. 3.- Color-Color diagram for the first and second emission episodes of GRB 980125 (BATSE trigger 6581). 


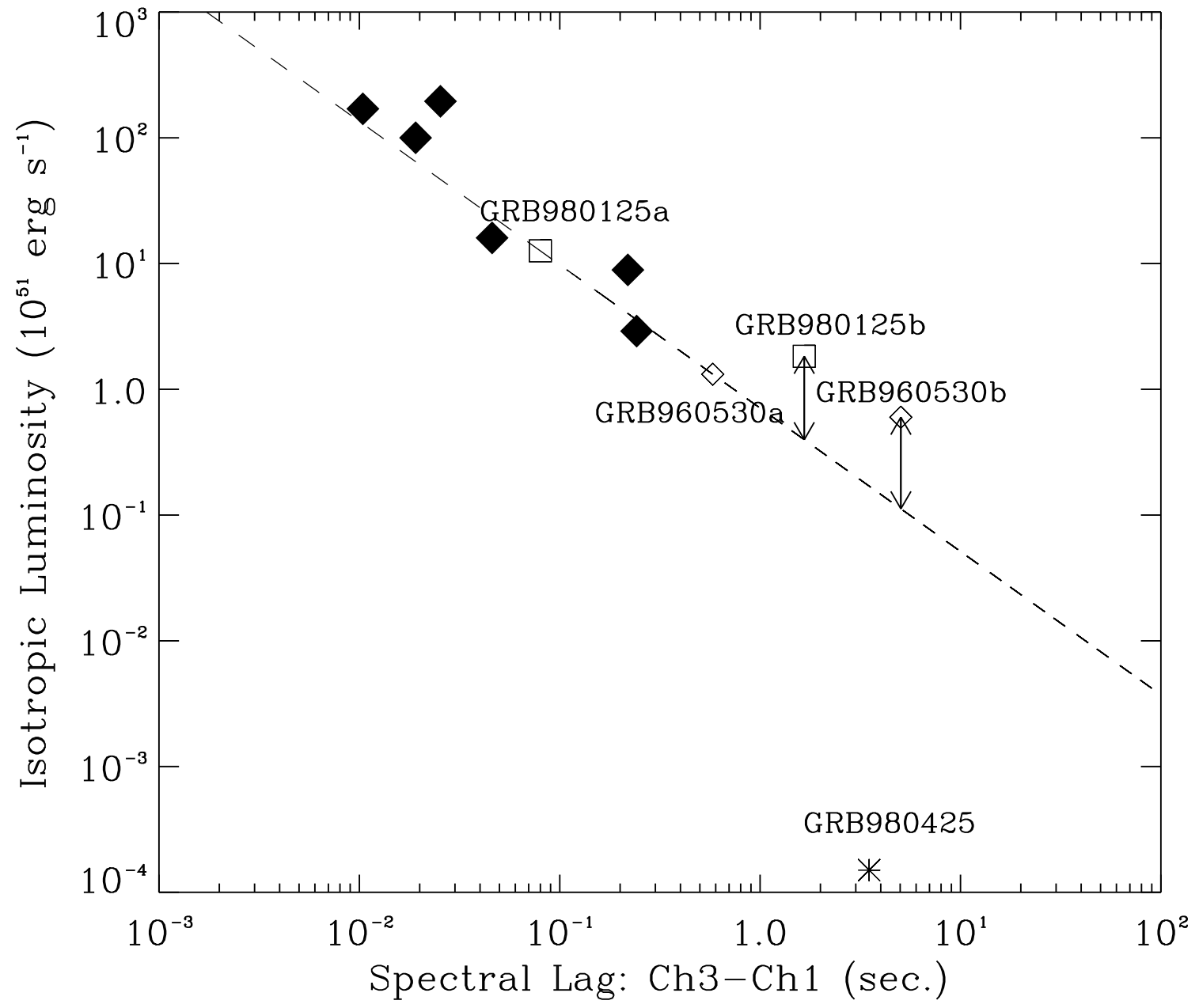

Fig. 4.- The lag vs. peak luminosity relation applied to GRBs 960530 and 980125). 
Table 1. Properties of Quiescent GRB 960530 (BATSE trigger 5478).

\begin{tabular}{lccccc}
\hline \hline Time Period & morphology & ILF $\alpha$ & Ch 21 lag (sec) & Ch 31 lag (sec) & Ch 32 lag (sec) \\
\hline before 200 sec & spiky FRED & $0.9 \pm 0.4$ & $0.1 \pm 0.11$ & $0.58 \pm 0.12$ & $0.58 \pm 0.04$ \\
after 200 sec & smooth gradual & $-7.5 \pm 1.2$ & $1.17 \pm 0.26$ & $5.04 \pm 0.06$ & $2.77 \pm 0.06$ \\
\hline
\end{tabular}


Table 2. Properties of Quiescent GRB 980125 (BATSE Trigger 6581).

\begin{tabular}{lccccc}
\hline \hline Time Period & morphology & ILF $\alpha$ & Ch 21 lag (sec) & Ch 31 lag (sec) & Ch 32 lag (sec) \\
\hline before 150 sec & complex + FRED & $-1.3 \pm 0.5$ & $0.04 \pm 0.02$ & $0.08 \pm 0.01$ & $0.10 \pm 0.01$ \\
150 to 250 sec & smooth gradual & $-3.1 \pm 0.5$ & $0.43 \pm 0.32$ & $1.66 \pm 0.19$ & $0.90 \pm 0.40$ \\
after 250 sec & smooth FRED & $-3.9 \pm 0.4$ & $0.07 \pm 0.03$ & $0.13 \pm 0.08$ & $0.05 \pm 0.08$ \\
\hline
\end{tabular}

